
enhancement by a novel statistical approach

4 Cheng $W_{u^{1,2}}$, Dui $W_{u^{1,2,3}}$, Jian Zhen $Y_{u^{4,5,6}}$

5

6

[1] Institute of Mass Spectrometer and Atmospheric Environment, Jinan University, Guangzhou 510632, China

[2] Guangdong Provincial Engineering Research Center for on-line source apportionment system of air pollution, Guangzhou 510632, China

[3] Institute of Tropical and Marine Meteorology, China Meteorological Administration, Guangzhou 510080, China

[4] Division of Environment, Hong Kong University of Science and Technology, Clear Water Bay, Hong Kong, China

[5] Atmospheric Research Centre, Fok Ying Tung Graduate School, Hong Kong University of Science and Technology, Nansha, China

[6] Department of Chemistry, Hong Kong University of Science and Technology, Clear Water Bay, Hong Kong, China

Corresponding to: Cheng Wu (wucheng.vip@,foxmail.com) and Jian Zhen Yu (jian.yu@ust.hk) 
This SI contains five tables and nineteen figures.

\section{Uncertainty of $\mathbf{E}_{\text {abs }}$ estimation}

The uncertainty of Eabs estimation depends on uncertainty propagation from MAE uncertainty, which can be calculated from (Harris, 2010):

$$
\begin{gathered}
M A E_{U n c}=M A E \times \sqrt{\left(\frac{\sigma_{a b s, U n c}}{\sigma_{a b s}}\right)^{2}+\left(\frac{E C_{U n c}}{E C}\right)^{2}} \\
E_{a b s, U n c}=E_{a b s} \times \sqrt{\left(\frac{M A E_{U n c}}{M A E}\right)^{2}+\left(\frac{M A E_{p, U n c}}{M A E_{p}}\right)^{2}}
\end{gathered}
$$

\section{Descriptions of customized programs used in this study for data analysis and}

\section{visualization}

Several computer programs were developed to meet specific research purpose in this study. All the programs are based on Igor Pro (www.wavemetrics.com) that provides a friendly GUI. Brief descriptions are given below.

\section{MRS program (Igor Pro based)}

The program (Figure S15) is written in Igro Pro (WaveMetrics, Inc. Lake Oswego, OR, USA) to feasible MRS calculation via a user-friendly GUI. The MRS application is not limited in SOC estimation, but can also be extended to other applications (e.g. Eabs estimation) as long as a reliable tracer is available.

MRS calculation can be done by different temporal cycles (batch calculation): by year, by year\&season, by season, by year\&month, by month, by year\&month\&hour. Data filter is also available to calculate MRS on a specific subset of data.

The program is available from $\underline{\mathrm{https}}$ //sites.google.com/site/wuchengust.

\section{Mie program and source code written in Igor Pro}

A computer program (Figure S16) written in Igro Pro (WaveMetrics, Inc. Lake Oswego, OR, USA) for Mie scattering calculation. Both BHMIE and BHCOAT (coated particles) algorithms(Bohren and Huffman, 1983) are included. The program is also capable of batch calculation for both algorithms. Available from https://sites.google.com/site/wuchengust. 


\section{Aethalometer data processing program (Igor Pro based)}

53 This handy tool (Figure S17) can perform different corrections (e.g. Weingartner, Virkkula) on 54 Aethalometer data. Raw Aethalometer data suffers from several artifacts including filter matrix 55 effect (multiple scattering), loading effect (shadowing) and scattering effect. Careful 56 corrections are needed for reporting light absorption coefficient from attenuation measurement. 57 This Igor based program can directly import Aethalometer raw data and perform corrections 58 (algorithm can be selected by user). Results can be exported to .csv files. Extra information 59 including statistics of sensor voltage from each channel, sampling flow rate, etc are plotted for 60 a quick QA/QC check. Available from https://sites.google.com/site/wuchengust.

61

\section{Histbox program (Igor Pro based)}

63 A handy tool (Figure S18) to generate histogram and box plots with many powerful features. 64 Data can be sorted by different time scale and batch plotting is available. Available from 65 https://sites.google.com/site/wuchengust.

66

\section{Scatter plot program}

68 Scatter plot (Figure S19) is a handy tool to maximize the efficiency of data visualization in 69 atmospheric science. The program includes Deming, WODR and York algorithm for linear 70 regression, which consider uncertainties in both $\mathrm{X}$ and $\mathrm{Y}$, that is more realistic for atmospheric applications. It is Igor based, and packed with lots of useful features for data analysis and graph plotting, including batch plotting, data masking via GUI, color coding in $\mathrm{Z}$ axis, data filtering and grouping. Available from https://sites.google.com/site/wuchengust. 


\section{Reference}

Andreae, M. O., Schmid, O., Yang, H., Chand, D., Yu, J. Z., Zeng, L. M., and Zhang, Y. H.: Optical properties and chemical composition of the atmospheric aerosol in urban Guangzhou, China, Atmos. Environ., 42, 6335-6350, doi: 10.1016/j.atmosenv.2008.01.030, 2008.

Bohren, C. F. and Huffman, D. R.: Absorption and scattering of light by small particles, Wiley, New York, xiv, 530 p. pp., 1983.

Chan, T. W., Brook, J. R., Smallwood, G. J., and Lu, G.: Time-resolved measurements of black carbon light absorption enhancement in urban and near-urban locations of southern Ontario, Canada, Atmos. Chem. Phys., 11, 10407-10432, 2011.

Chow, J. C., Watson, J. G., Doraiswamy, P., Chen, L. W. A., Sodeman, D. A., Lowenthal, D. H., Park, K., Arnott, W. P., and Motallebi, N.: Aerosol light absorption, black carbon, and elemental carbon at the Fresno Supersite, California, Atmos Res, 93, 874-887, doi: DOI 10.1016/j.atmosres.2009.04.010, 2009.

Chuang, P. Y., Duvall, R. M., Bae, M. S., Jefferson, A., Schauer, J. J., Yang, H., Yu, J. Z., and Kim, J.: Observations of elemental carbon and absorption during ACE-Asia and implications for aerosol radiative properties and climate forcing, J. Geophys. Res., 108, 8634, doi: Doi 10.1029/2002jd003254, 2003.

Doran, J. C., Barnard, J. C., Arnott, W. P., Cary, R., Coulter, R., Fast, J. D., Kassianov, E. I., Kleinman, L., Laulainen, N. S., Martin, T., Paredes-Miranda, G., Pekour, M. S., Shaw, W. J., Smith, D. F., Springston, S. R., and Yu, X. Y.: The T1-T2 study: evolution of aerosol properties downwind of Mexico City, Atmos. Chem. Phys., 7, 1585-1598, doi: 10.5194/acp-7-1585-2007, 2007.

Harris, D. C.: Quantitative chemical analysis, 8th ed., W.H. Freeman and Co., New York, 2010. Knox, A., Evans, G. J., Brook, J. R., Yao, X., Jeong, C. H., Godri, K. J., Sabaliauskas, K., and Slowik, J. G.: Mass Absorption Cross-Section of Ambient Black Carbon Aerosol in Relation to Chemical Age, Aerosol. Sci. Technol., 43, 522-532, doi: Doi 10.1080/02786820902777207, 2009.

Lack, D. A. and Cappa, C. D.: Impact of brown and clear carbon on light absorption enhancement, single scatter albedo and absorption wavelength dependence of black carbon, Atmos. Chem. Phys., 10, 4207-4220, doi: DOI 10.5194/acp-10-4207-2010, 2010.

Lan, Z.-J., Huang, X.-F., Yu, K.-Y., Sun, T.-L., Zeng, L.-W., and Hu, M.: Light absorption of black carbon aerosol and its enhancement by mixing state in an urban atmosphere in South China, Atmos. Environ., 69, 118-123, doi: http://dx.doi.org/10.1016/j.atmosenv.2012.12.009, 2013.

Liu, D., Flynn, M., Gysel, M., Targino, A., Crawford, I., Bower, K., Choularton, T., Jurányi, Z., Steinbacher, M., Hüglin, C., Curtius, J., Kampus, M., Petzold, A., Weingartner, E., Baltensperger, U., and Coe, H.: Single particle characterization of black carbon aerosols at a tropospheric alpine site in Switzerland, Atmos. Chem. Phys., 10, 7389-7407, doi: 10.5194/acp10-7389-2010, 2010.

Mayol-Bracero, O. L., Gabriel, R., Andreae, M. O., Kirchstetter, T. W., Novakov, T., Ogren, J., Sheridan, P., and Streets, D. G.: Carbonaceous aerosols over the Indian Ocean during the Indian Ocean Experiment (INDOEX): Chemical characterization, optical properties, and probable sources, J. Geophys. Res., 107, 8030, doi: Doi 10.1029/2000jd000039, 2002.

Moosmuller, H., Chakrabarty, R. K., Ehlers, K. M., and Arnott, W. P.: Absorption Angstrom coefficient, brown carbon, and aerosols: basic concepts, bulk matter, and spherical particles, Atmos. Chem. Phys., 11, 1217-1225, doi: DOI 10.5194/acp-11-1217-2011, 2011.

Pandolfi, M., Cusack, M., Alastuey, A., and Querol, X.: Variability of aerosol optical properties in the Western Mediterranean Basin, Atmos. Chem. Phys., 11, 8189-8203, doi: DOI 10.5194/acp-11-8189-2011, 2011. 
Thompson, J. E., Hayes, P. L., Jimenez, K. A. J. L., Zhang, X., Liu, J., Weber, R. J., and Buseck,

126 P. R.: Aerosol Optical Properties at Pasadena, CA During CalNex 2010, Atmos Environ, doi:

127 10.1016/j.atmosenv.2012.03.011, 2012.

128 Wang, Q., Huang, R., Zhao, Z., Cao, J., Ni, H., Tie, X., Zhu, C., Shen, Z., Wang, M., and Dai, 129 W.: Effects of photochemical oxidation on the mixing state and light absorption of black carbon 130 in the urban atmosphere of China, Environmental Research Letters, 12, 044012, 2017.

131 Wang, Q. Y., Huang, R. J., Cao, J. J., Han, Y. M., Wang, G. H., Li, G. H., Wang, Y. C., Dai, 132 W. T., Zhang, R. J., and Zhou, Y. Q.: Mixing State of Black Carbon Aerosol in a Heavily 133 Polluted Urban Area of China: Implications for Light Absorption Enhancement, Aerosol. Sci. 134 Technol., 48, 689-697, doi: 10.1080/02786826.2014.917758, 2014.

135 Xu, J., Bergin, M. H., Yu, X., Liu, G., Zhao, J., Carrico, C. M., and Baumann, K.: Measurement 136 of aerosol chemical, physical and radiative properties in the Yangtze delta region of China, 137 Atmos. Environ., 36, 161-173, 2002.

138 Yang, M., Howell, S. G., Zhuang, J., and Huebert, B. J.: Attribution of aerosol light absorption 139 to black carbon, brown carbon, and dust in China - interpretations of atmospheric measurements 140 during EAST-AIRE, Atmos. Chem. Phys., 9, 2035-2050, 2009. 
Table S1. Comparison of Mass absorption efficiency (MAE) at various locations. For literature MAE values at different wavelengths rather than 550nm, an estimated $\mathrm{MAE}_{550}$ is given in the brackets following equations given by Moosmuller et al. (2011) assuming AAE of 1.

\begin{tabular}{|c|c|c|c|c|c|c|c|c|c|c|c|c|}
\hline & & & & & & & & & ctimod & observed MAE & $\left.{ }^{2} \mathrm{~g}^{-1}\right)$ & \\
\hline Guangzhou, China & Suburban & $2012.2-2013.1$ & $\mathrm{PM}_{2.5}$ & 550 & $\mathrm{AE}$ & NIOSH_TOT & $42.20 \pm 29.41$ & $2.63 \pm 2.27$ & $13^{*}$ & $19.02 \pm 6.60$ & 16.16 & This study \\
\hline Shenzhen, China & Urban & 2011.8-9 & $\mathrm{PM}_{2.5}$ & 532 & PAS & LII & $25.4 \pm 19.0$ & $4.0 \pm 3.1$ & I & $6.5 \pm 0.5[6.29 \pm 0.48]$ & / & (Lan et al., 2013) \\
\hline Xi'an, China & Urban & $2012.12-2013.1$ & $\mathrm{PM}_{2.5}$ & 870 & PAS & LII & I & $8.8 \pm 7.3$ & $7.17[11.34]$ & I & $7.62[12.05]$ & (Wang et al., 2014) \\
\hline Xi'an, China & Urban & 2013.2 & $\mathrm{PM}_{2.5}$ & 532 & PAS & LII & & & & $14.6 \pm 5.6$ & 12.7 & (Wang et al., 2017) \\
\hline Guangzhou, China & Urban & 2004.10 & $\mathrm{PM}_{2.5}$ & 532 & PAS & NIOSH_TOT & $91 \pm 60$ & 7.1 & 7.7[7.44] & I & I & (Andreae et al., 2008) \\
\hline Fresno, USA & Urban & 2005.8-9 & $\mathrm{PM}_{2.5}$ & 532 & PAS & $\begin{array}{c}\text { IMPROVE_A_TOR } \\
\text { NIOSH_TOT }\end{array}$ & 5.06 & $\begin{array}{l}1.01 \\
0.58\end{array}$ & ' & $\begin{array}{c}6.1 \pm 2.5[5.9 \pm 2.42] \\
9.3 \pm 2.4[8.99 \pm 2.32]\end{array}$ & ' & (Chow et al., 2009) \\
\hline $\begin{array}{l}\text { T1,Mexico city, } \\
\text { Mexico } \\
\text { Pasadena, USA }\end{array}$ & $\begin{array}{c}\text { Suburban } \\
\text { Urban }\end{array}$ & $\begin{array}{c}2006.3 \\
2010.5-6\end{array}$ & $\begin{array}{l}\mathrm{PM}_{2.5} \\
\mathrm{PM}_{2.5}\end{array}$ & $\begin{array}{l}870 \\
532\end{array}$ & $\begin{array}{l}\text { PAS } \\
\text { AM }\end{array}$ & $\begin{array}{l}\text { NIOSH_TOT } \\
\text { NIOSH_tOT }\end{array}$ & $\begin{array}{c}/ \\
3.8 \pm 3.4\end{array}$ & $\begin{array}{c}/ \\
0.6 \sim 0.7\end{array}$ & $\begin{array}{c}/ \\
5.7[5.51]\end{array}$ & $\begin{array}{c}9.2 \sim 9.7^{* * *}[14.55 \sim 15.34] \\
\text { / }\end{array}$ & ' & $\begin{array}{l}\text { (Doran et al., 2007) } \\
\text { (Thompson et al., }\end{array}$ \\
\hline Toronto, Canada & Urban & 2006.12-2007.1 & $\mathrm{PM}_{2.5}$ & 760 & PAS & NIOSH_TOT & I & I & $\begin{array}{c}6.9 \sim 9.1^{* *} \\
{[9.53 \sim 12.57]}\end{array}$ & $9.3 \sim 9.9[12.85 \sim 13.68]$ & I & (Knox et al., 2009) \\
\hline Toronto, Canada & Suburban & & & & & & $3 \sim 6$ & $0.10 \sim 0.14$ & I & $30 \sim 43[42.6 \sim 61.06]$ & / & \\
\hline Windsor, Canada & Urban & 2007.8 & $\mathrm{PM}_{2.5}$ & 781 & PAS & LII & $4.4 \pm 2.9$ & $0.27 \pm 0.23$ & l & $16 \pm 1[22.72 \pm 1.42]$ & l & (Chan et al., 2011) \\
\hline Ottawa, Canada & Urban & & & & & & $26 \pm 17$ & $1.7 \pm 0.9$ & I & $15 \pm 3[21.3 \pm 4.26]$ & / & \\
\hline Beijing, China & Rural & 2005.3 & / & 550 & $\mathrm{AE}$ & NIOSH_TOT & l & l & 9.5 & 11.3 & I & (Yang et al., 2009) \\
\hline Montseny, Spin & $\begin{array}{c}\text { Rural } \\
\text { (Mediterranean ) }\end{array}$ & 2009.11-2010.10 & $\mathrm{PM}_{10}$ & 637 & MAAP & NIOSH_TOT & $2.8 \pm 2.2$ & l & $10.4[12.04]$ & I & / & (Pandolfi et al., 2011) \\
\hline $\begin{array}{c}\text { Jungfraujoch, } \\
\text { Switzerland }\end{array}$ & $\begin{array}{c}\text { Rural } \\
\text { (high alpine) }\end{array}$ & $2007.2-3$ & I & 637 & MAAP & LII & I & l & l & $10.2 \pm 3.2[11.81 \pm 3.71]$ & / & (Liu et al., 2010) \\
\hline Lin'an, China & Rural & 1999.11 & $\mathrm{PM}_{2.5}$ & 550 & PSAP & NIOSH_TOT & $23 \pm 14$ & $3.4 \pm 1.7$ & I & $8.6 \pm 7.0$ & I & (Xu et al., 2002) \\
\hline Jeju Island, Korea & $\begin{array}{l}\text { Coastal Rural, } \\
\text { (East China Sea) }\end{array}$ & 2001.4 & $\mathrm{PM}_{10}$ & 550 & PSAP & NIOSH_TOT & I & I & I & $12.6 \pm 2.6$ & I & (Chuang et al., 2003) \\
\hline Maldives & Oceanic rural & $1999.2-3$ & $\mathrm{PM}_{3}$ & 550 & PSAP & EGA & $62 \pm 34$ & $2.5 \pm 1.4$ & 6.6 & 8.1 & I & $\begin{array}{c}\text { (Mayol-Bracero et al., } \\
\text { 2002) }\end{array}$ \\
\hline
\end{tabular}

*Determined by Minimium R Squared method; ** Median values;

AE:Aethalometer ; PAS photo acoustic spectrometer; MAAP: Multi Angle Absorption Photometer; PSAP: particle soot absorption photometer; AM: albedo meter; LII: Laser induced incandescence 
Table S2. Statistics of monthly MAE550nm

\begin{tabular}{lllllllllll}
\hline Month & 95th & 75th & 50th & 25th & 5th & Mean & Max & Min & S.D. & N \\
\hline Feb-2012 & 31.29 & 22.04 & 18.12 & 15.74 & 13.90 & 19.72 & 47.73 & 8.50 & 5.78 & 533 \\
Mar-2012 & 28.33 & 19.80 & 17.48 & 15.92 & 13.77 & 18.78 & 45.56 & 10.98 & 4.92 & 663 \\
Apr-2012 & 33.06 & 22.66 & 18.24 & 16.11 & 13.85 & 20.21 & 48.29 & 6.01 & 6.23 & 595 \\
May-2012 & 33.74 & 23.35 & 19.61 & 17.17 & 14.73 & 21.22 & 48.40 & 6.33 & 5.99 & 533 \\
Jun-2012 & 39.73 & 27.17 & 21.76 & 18.72 & 15.04 & 23.70 & 49.07 & 5.62 & 7.37 & 333 \\
Jul-2012 & 35.96 & 24.62 & 19.12 & 15.64 & 12.71 & 21.14 & 49.63 & 9.23 & 7.65 & 609 \\
Aug-2012 & 42.94 & 27.99 & 22.01 & 16.24 & 12.55 & 23.50 & 49.95 & 9.75 & 8.98 & 556 \\
Sep-2012 & 33.15 & 21.11 & 17.61 & 15.25 & 12.99 & 19.31 & 49.54 & 10.39 & 6.18 & 684 \\
Oct-2012 & 20.72 & 15.84 & 13.95 & 12.60 & 11.18 & 14.70 & 34.09 & 7.34 & 3.21 & 715 \\
Nov-2012 & 28.64 & 18.67 & 15.53 & 13.72 & 11.95 & 17.18 & 48.41 & 8.34 & 5.58 & 506 \\
Dec-2012 & 29.92 & 19.32 & 15.74 & 13.67 & 11.78 & 17.39 & 48.73 & 9.33 & 5.86 & 591 \\
Jan-2013 & 21.60 & 16.24 & 14.48 & 13.03 & 11.79 & 15.33 & 48.48 & 7.16 & 3.97 & 709 \\
\hline
\end{tabular}

Table S3. Statistics of monthly AAE470-660

\begin{tabular}{lcccccccccc}
\hline Month & 95th & 75th & 50th & 25th & 5th & Mean & Max & Min & S.D. & N \\
\hline Feb-2012 & 1.41 & 1.25 & 1.17 & 1.09 & 0.94 & 1.17 & 1.70 & 0.78 & 0.14 & 533 \\
Mar-2012 & 1.31 & 1.17 & 1.08 & 1.00 & 0.92 & 1.09 & 1.45 & 0.68 & 0.12 & 663 \\
Apr-2012 & 1.16 & 1.07 & 1.01 & 0.95 & 0.83 & 1.00 & 1.35 & 0.56 & 0.10 & 587 \\
May-2012 & 1.11 & 1.03 & 0.99 & 0.93 & 0.85 & 0.98 & 1.21 & 0.50 & 0.09 & 530 \\
Jun-2012 & 1.17 & 1.09 & 1.03 & 0.97 & 0.88 & 1.02 & 1.29 & 0.39 & 0.10 & 333 \\
Jul-2012 & 1.19 & 1.09 & 1.03 & 0.97 & 0.84 & 1.03 & 1.38 & 0.47 & 0.11 & 604 \\
Aug-2012 & 1.16 & 1.08 & 1.03 & 0.97 & 0.88 & 1.02 & 1.30 & 0.64 & 0.08 & 556 \\
Sep-2012 & 1.21 & 1.11 & 1.04 & 0.98 & 0.90 & 1.05 & 1.37 & 0.68 & 0.10 & 684 \\
Oct-2012 & 1.25 & 1.15 & 1.07 & 1.01 & 0.93 & 1.08 & 1.36 & 0.84 & 0.10 & 715 \\
Nov-2012 & 1.22 & 1.14 & 1.09 & 1.03 & 0.97 & 1.09 & 1.45 & 0.88 & 0.08 & 506 \\
Dec-2012 & 1.31 & 1.21 & 1.15 & 1.09 & 0.99 & 1.15 & 1.41 & 0.92 & 0.09 & 591 \\
Jan-2013 & 1.36 & 1.25 & 1.17 & 1.11 & 1.00 & 1.18 & 1.63 & 0.90 & 0.11 & 709 \\
\hline
\end{tabular}


Table S4. Statistics of monthly SSA

\begin{tabular}{lcccccccccc}
\hline Month & 95th & 75th & 50th & 25th & 5th & Mean & Max & Min & S.D. & N \\
\hline Feb-2012 & 0.91 & 0.89 & 0.87 & 0.84 & 0.79 & 0.86 & 0.94 & 0.65 & 0.04 & 530 \\
Mar-2012 & 0.91 & 0.89 & 0.86 & 0.83 & 0.77 & 0.85 & 0.95 & 0.42 & 0.05 & 660 \\
Apr-2012 & 0.92 & 0.89 & 0.86 & 0.83 & 0.76 & 0.85 & 0.94 & 0.45 & 0.06 & 552 \\
May-2012 & 0.92 & 0.90 & 0.87 & 0.83 & 0.74 & 0.85 & 0.94 & 0.45 & 0.06 & 532 \\
Jun-2012 & 0.92 & 0.89 & 0.86 & 0.81 & 0.74 & 0.85 & 0.95 & 0.64 & 0.06 & 328 \\
Jul-2012 & 0.91 & 0.87 & 0.83 & 0.79 & 0.71 & 0.83 & 0.95 & 0.57 & 0.06 & 602 \\
Aug-2012 & 0.94 & 0.92 & 0.89 & 0.85 & 0.79 & 0.88 & 0.96 & 0.67 & 0.05 & 547 \\
Sep-2012 & 0.94 & 0.91 & 0.88 & 0.84 & 0.75 & 0.87 & 0.96 & 0.55 & 0.06 & 682 \\
Oct-2012 & 0.94 & 0.93 & 0.91 & 0.89 & 0.84 & 0.90 & 0.96 & 0.66 & 0.03 & 715 \\
Nov-2012 & 0.91 & 0.89 & 0.87 & 0.83 & 0.75 & 0.85 & 0.94 & 0.18 & 0.06 & 506 \\
Dec-2012 & 0.91 & 0.89 & 0.86 & 0.82 & 0.74 & 0.85 & 0.94 & 0.66 & 0.05 & 591 \\
Jan-2013 & 0.91 & 0.89 & 0.87 & 0.85 & 0.79 & 0.86 & 0.93 & 0.64 & 0.04 & 709 \\
\hline
\end{tabular}

Table S5. Statistics of monthly Eabs550

\begin{tabular}{lcccccccccc}
\hline Month & 95th & 75th & 50th & 25th & 5th & Mean & Max & Min & S.D. & N \\
\hline Feb-2012 & 2.24 & 1.57 & 1.29 & 1.12 & 0.99 & 1.41 & 3.41 & 0.61 & 0.41 & 533 \\
Mar-2012 & 1.76 & 1.23 & 1.09 & 0.99 & 0.86 & 1.17 & 2.83 & 0.68 & 0.31 & 663 \\
Apr-2012 & 2.45 & 1.68 & 1.35 & 1.19 & 1.03 & 1.50 & 3.58 & 0.44 & 0.46 & 595 \\
May-2012 & 2.50 & 1.73 & 1.45 & 1.27 & 1.09 & 1.57 & 3.58 & 0.47 & 0.44 & 533 \\
Jun-2012 & 2.74 & 1.87 & 1.50 & 1.29 & 1.04 & 1.63 & 3.38 & 0.39 & 0.51 & 333 \\
Jul-2012 & 2.95 & 2.02 & 1.57 & 1.28 & 1.04 & 1.73 & 4.07 & 0.76 & 0.63 & 609 \\
Aug-2012 & 3.61 & 2.35 & 1.85 & 1.36 & 1.05 & 1.97 & 4.20 & 0.82 & 0.75 & 556 \\
Sep-2012 & 2.53 & 1.61 & 1.34 & 1.16 & 0.99 & 1.47 & 3.78 & 0.79 & 0.47 & 684 \\
Oct-2012 & 1.87 & 1.43 & 1.26 & 1.14 & 1.01 & 1.32 & 3.07 & 0.66 & 0.29 & 715 \\
Nov-2012 & 2.31 & 1.51 & 1.25 & 1.11 & 0.96 & 1.39 & 3.90 & 0.67 & 0.45 & 506 \\
Dec-2012 & 2.54 & 1.64 & 1.33 & 1.16 & 1.00 & 1.47 & 4.13 & 0.79 & 0.50 & 591 \\
Jan-2013 & 1.83 & 1.38 & 1.23 & 1.10 & 1.00 & 1.30 & 4.11 & 0.61 & 0.34 & 709 \\
\hline
\end{tabular}




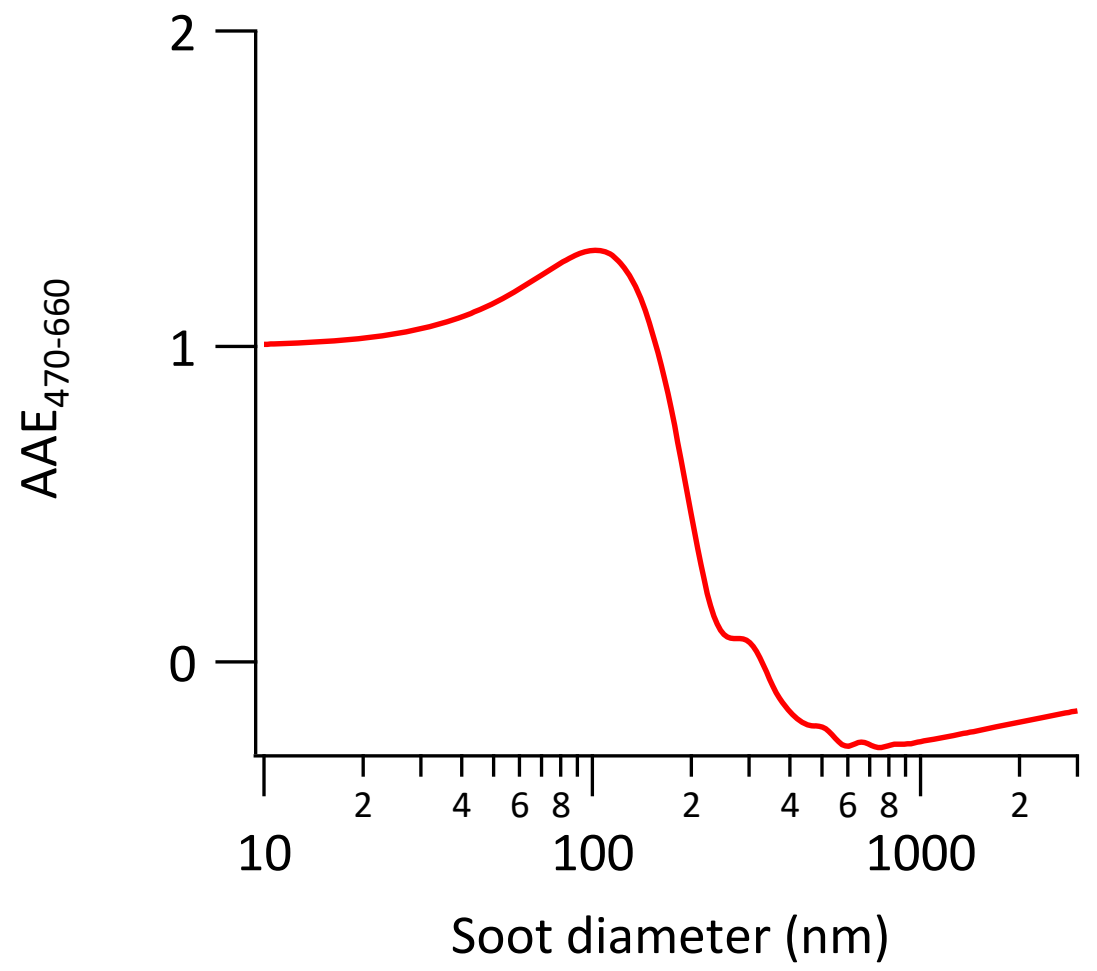

Figure $\mathrm{S} 1$. Mie simulated $A A E_{470-660}$ of a bare soot particle as a function of diameter with a Refractive index of $1.85-0.71$. 

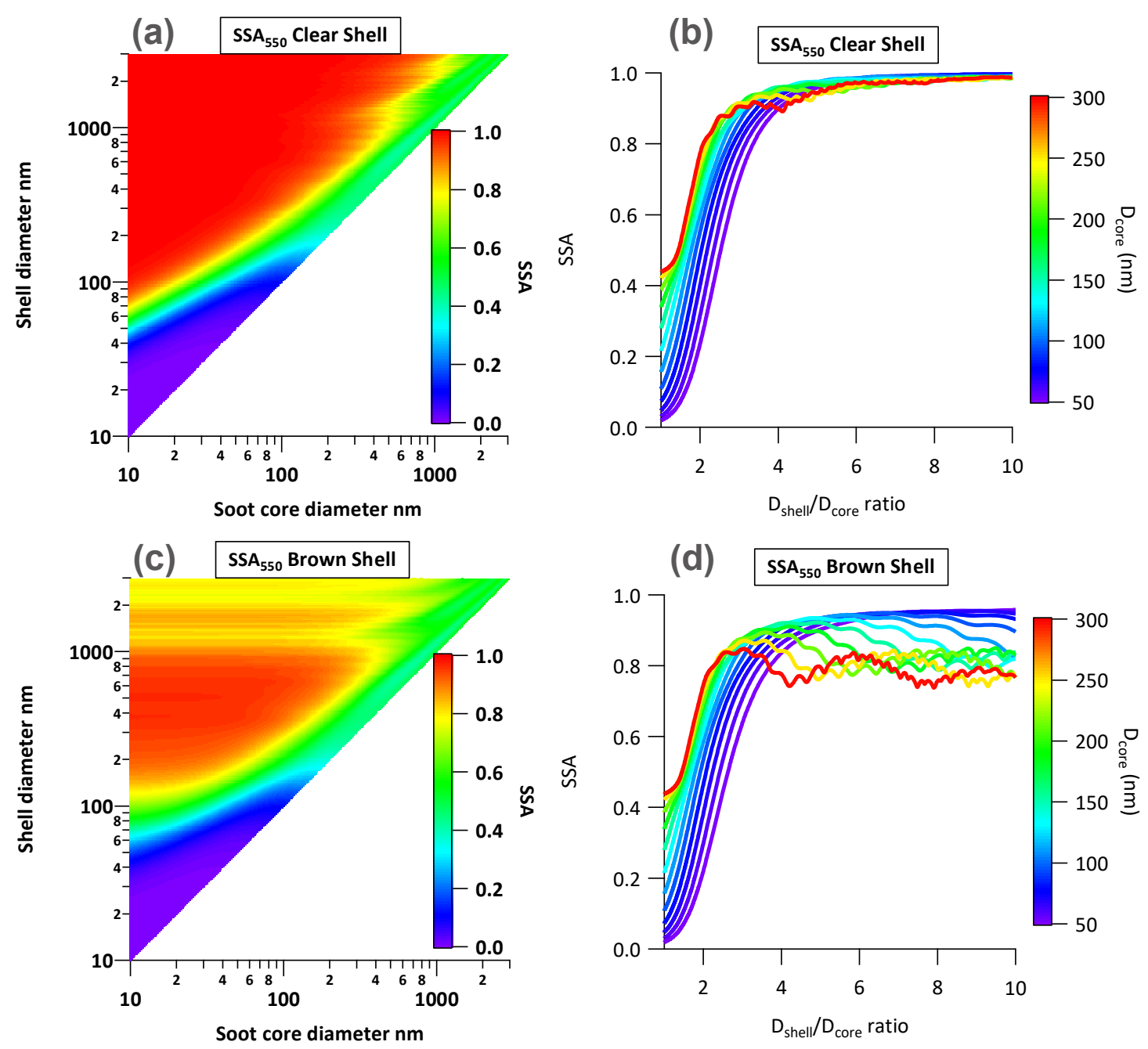

Figure S2. Mie simulated size dependency of soot particles SSA at wavelength $550 \mathrm{~nm}$. (a)Combination of different shell ( $y$ axis) and core diameters ( $x$ axis). The color coding represents the SSA of a particle with specific core and shell size; (b) Cross-sections views of (a). The color coding represents different $D_{\text {core }}$ in the range of $50-300 \mathrm{~nm}$. (c)Combination of different shell (y axis) and core diameters ( $x$ axis). The color coding represents the Eabs of a particle with specific core and shell size; (d) Cross-sections views of (c). The color coding represents different diameters of soot core in the range of $50-300 \mathrm{~nm}$. 


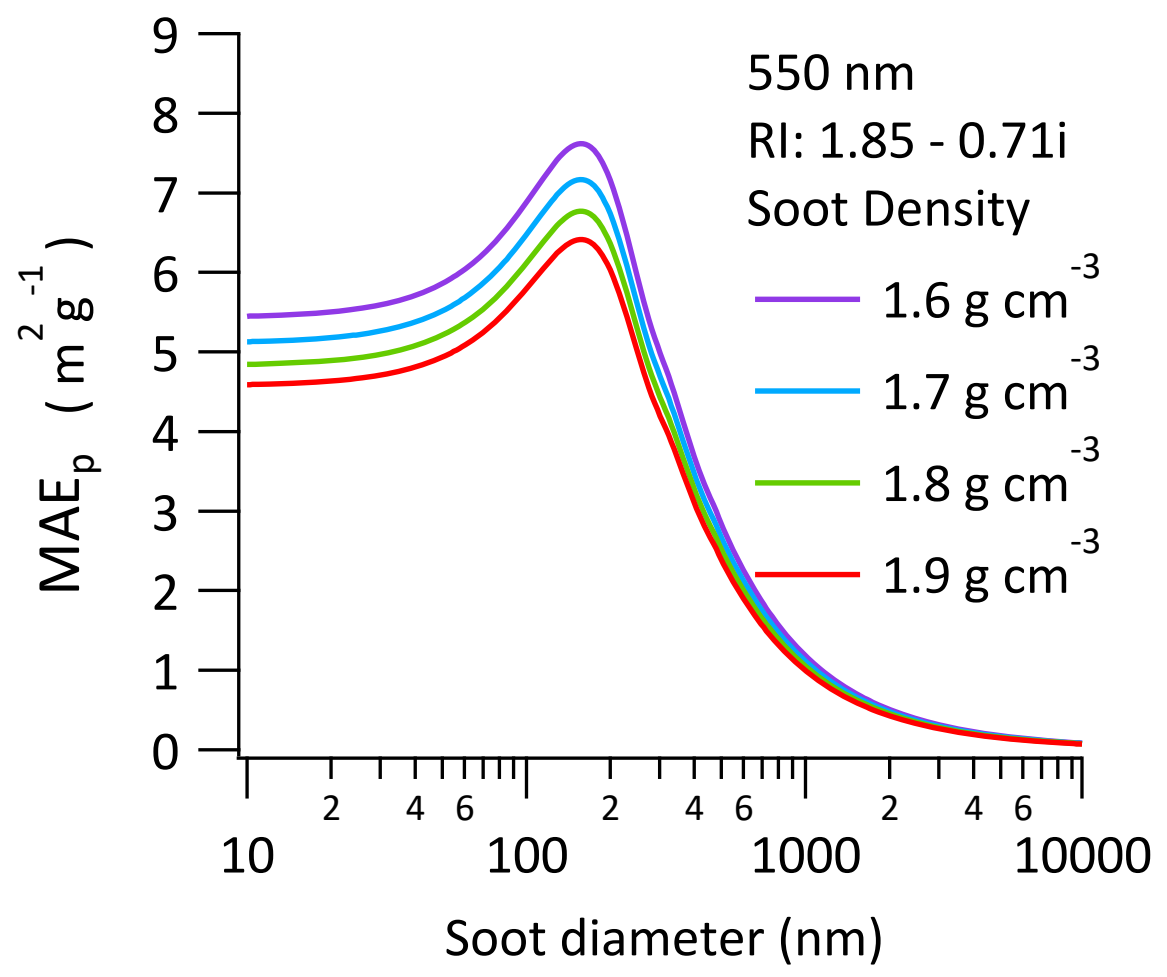

Figure S3. Mie simulated mass absorption efficiency (MAE $)$ of a bare soot particle as a function of diameter at a wavelength of $550 \mathrm{~nm}$. Refractive index is $1.85-0.71 \mathrm{i}$ and density varied from 1.6 to $1.9 \mathrm{~g} \mathrm{~cm}^{-3}$. 

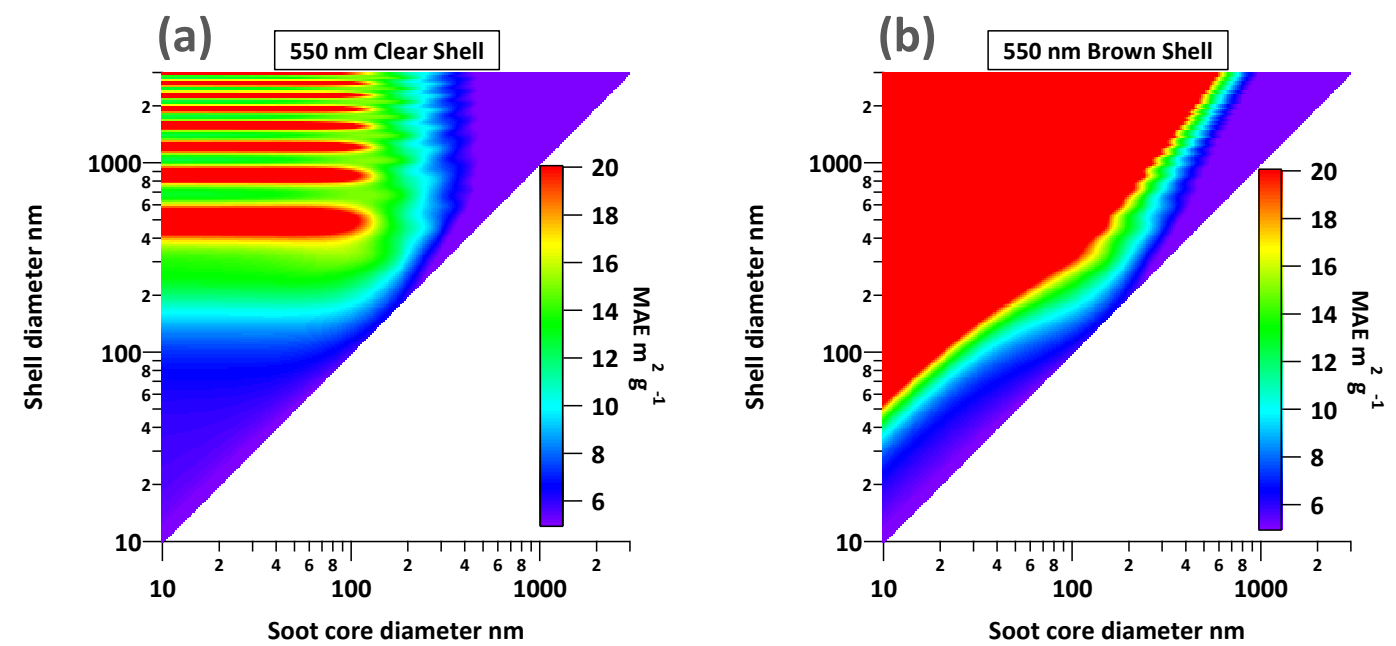

Figure S4 Mie simulated mass absorption efficiency (MAE) of a bare soot particle as a function of diameter at a wavelength of $550 \mathrm{~nm}$. Refractive index is $1.85-0.71 \mathrm{i}$ and density is $1.9 \mathrm{~g} \mathrm{~cm}^{-}$ ${ }^{3}$ for soot core. Refractive index for clear coating is 1.55 . Refractive index for brown coating is wavelength dependent adopted from Lack and Cappa (Lack and Cappa, 2010). 

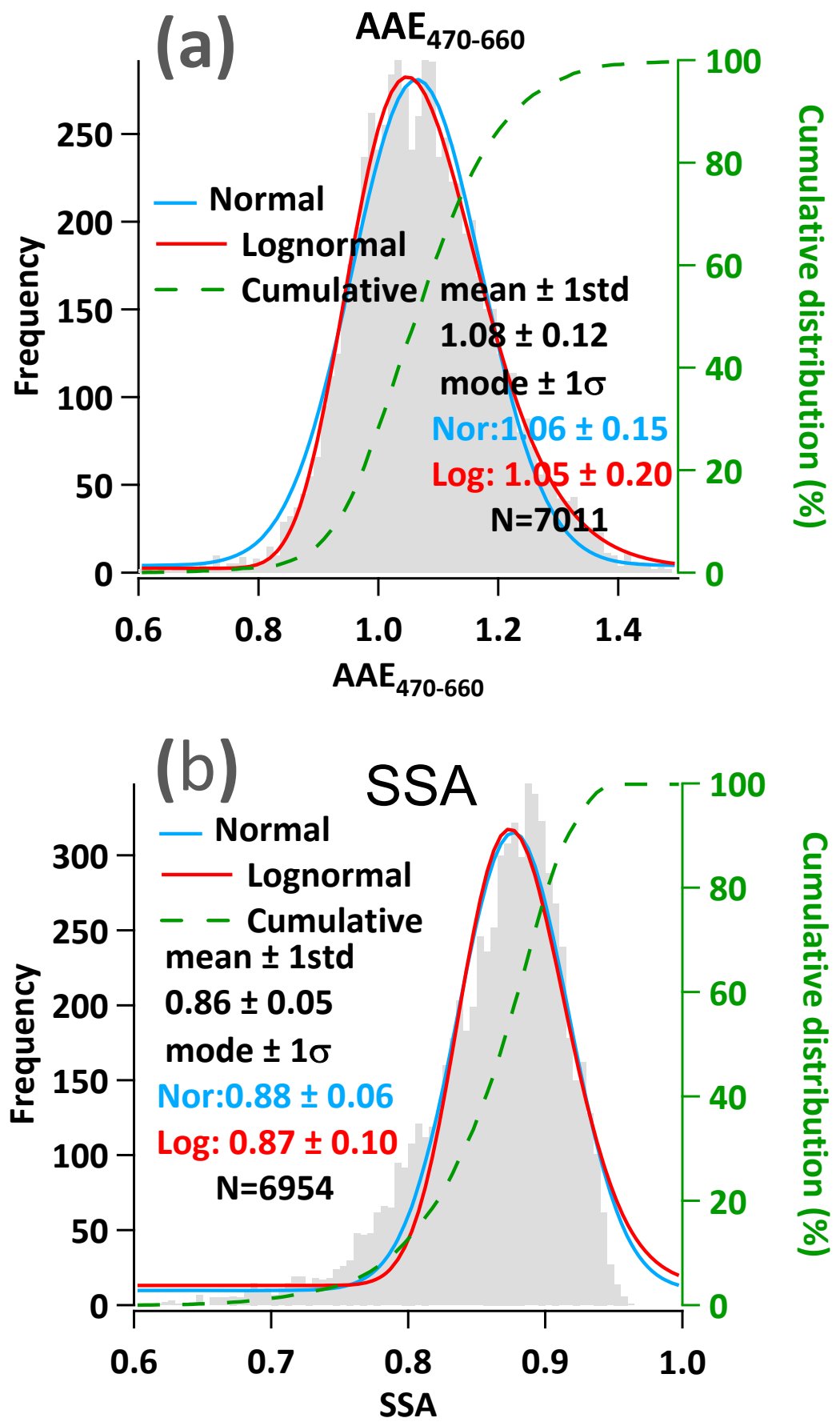

Figure S5. Measured annual statistics of $A A E_{470-660}$, and SSA. (a) Annual frequency distribution of AAE at $550 \mathrm{~nm}$. (b) Annual frequency distribution of SSA. The blue and red line represent normal and lognormal fitting curve respectively. 


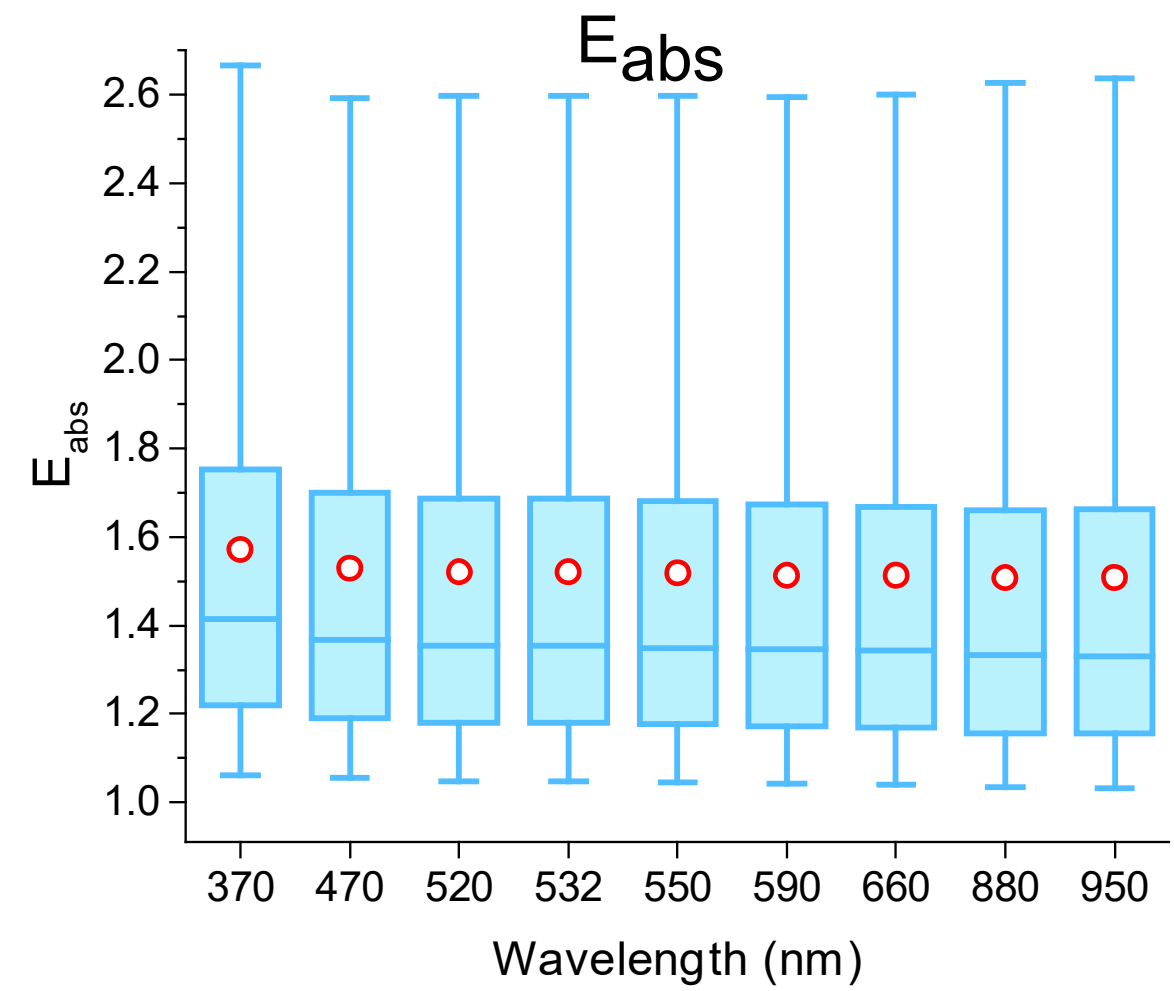

\begin{tabular}{cccccccccc} 
Wavelength $(\mathrm{nm})$ & 370 & 470 & 520 & 532 & 550 & 590 & 660 & 880 & 950 \\
\hline $\mathrm{E}_{\text {abs }}$ mean & 1.57 & 1.53 & 1.52 & 1.52 & 1.52 & 1.51 & 1.51 & 1.51 & 1.51 \\
$\mathrm{E}_{\text {abs }}$ S.D. & 0.52 & 0.51 & 0.51 & 0.51 & 0.51 & 0.51 & 0.52 & 0.53 & 0.53
\end{tabular}

Figure S6. Spectrum annual average Eabs from 370 to $950 \mathrm{~nm}$. 


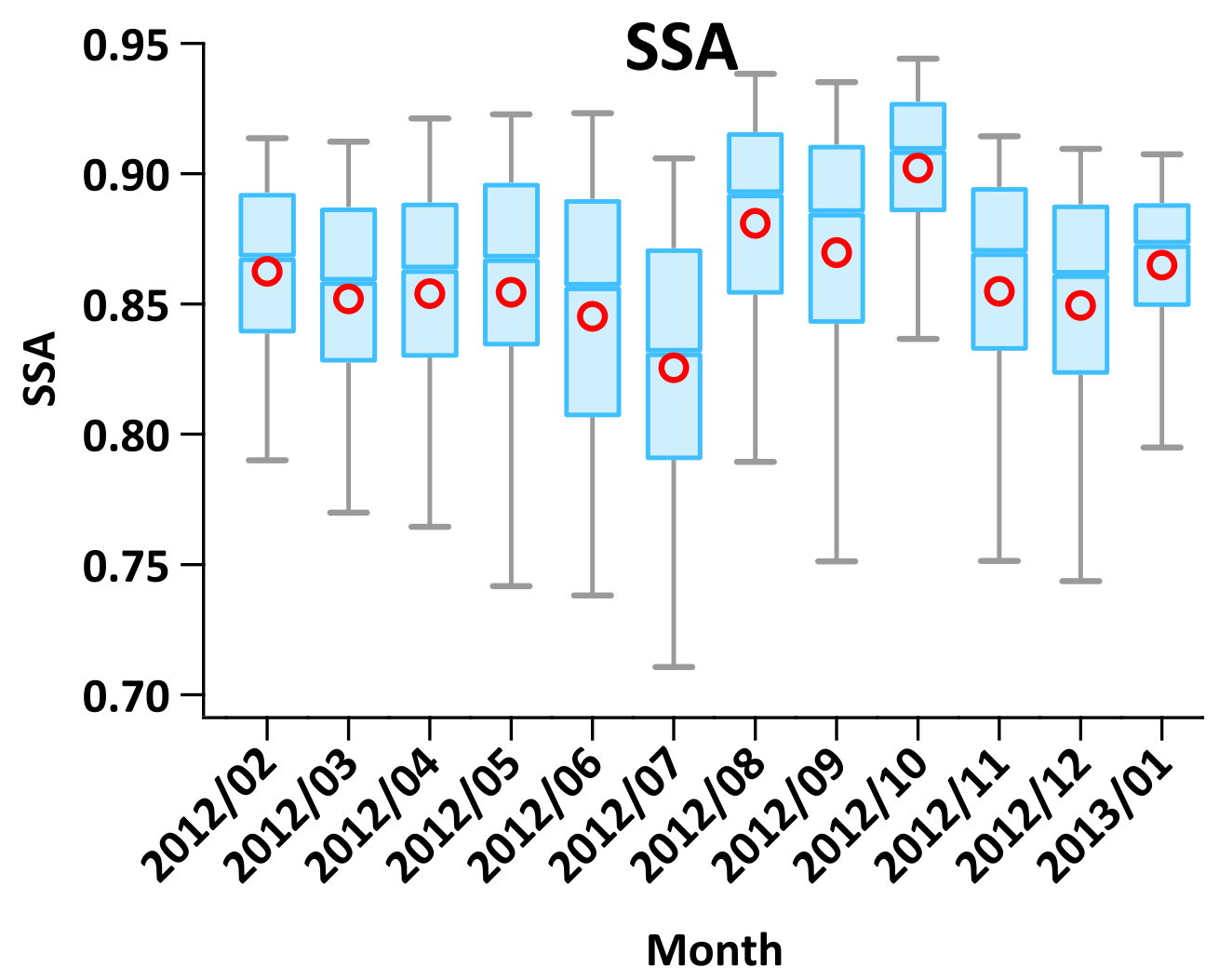

Figure S7. Measured monthly variations of SSA. 


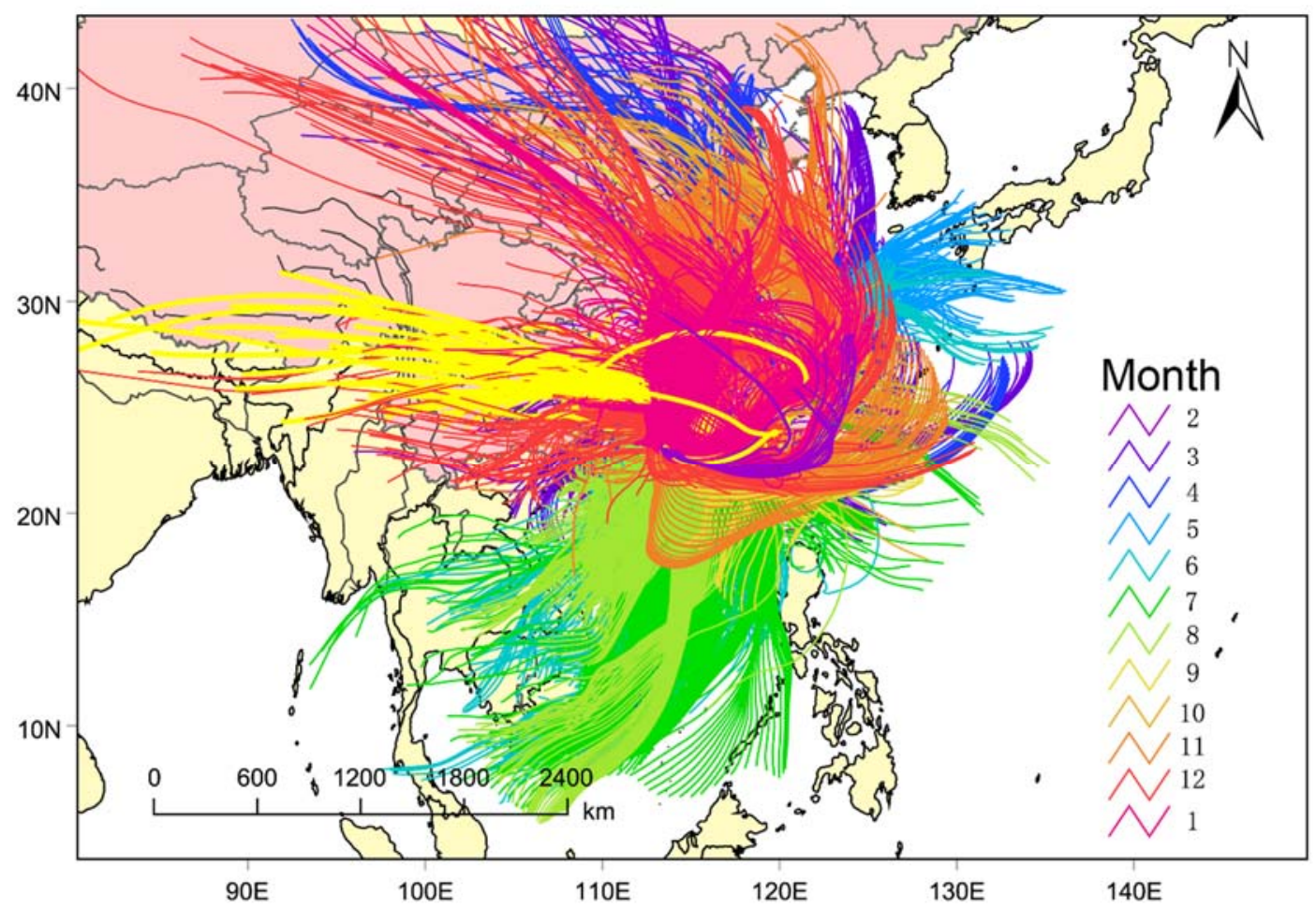

Figure S8. Hourly back trajectories for the past 72 hours calculated using NOAA's HYSPLIT model from Feb 2012 to Jan 2013. The color coding represents different months.

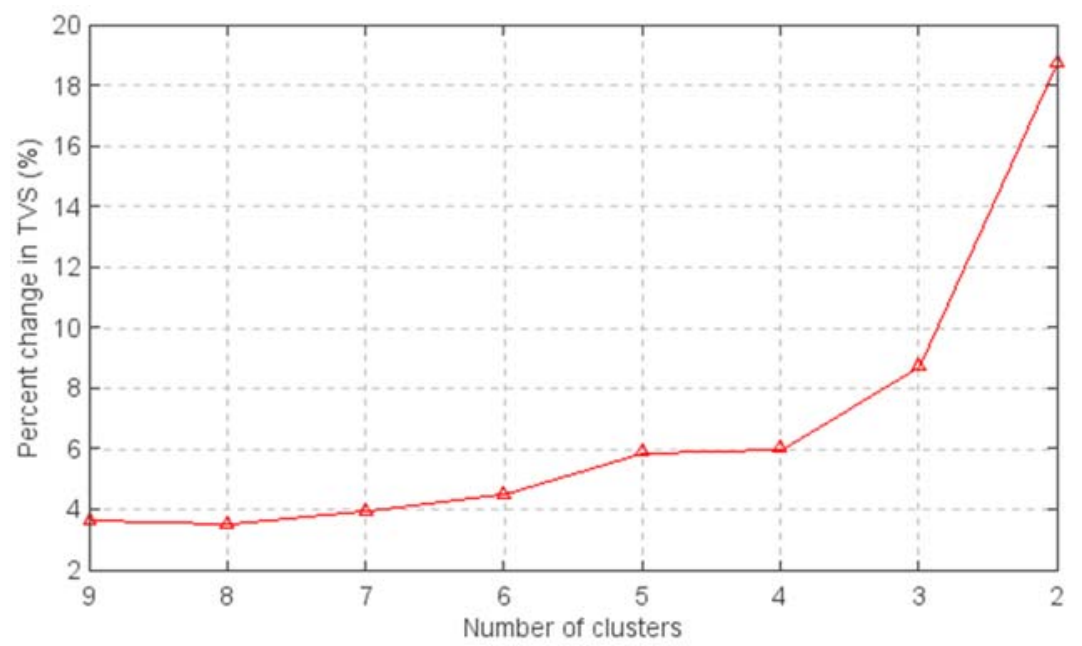

Figure S9. Total spatial variance (TSV) as a function of number of clusters in back trajectories clustering analysis. 

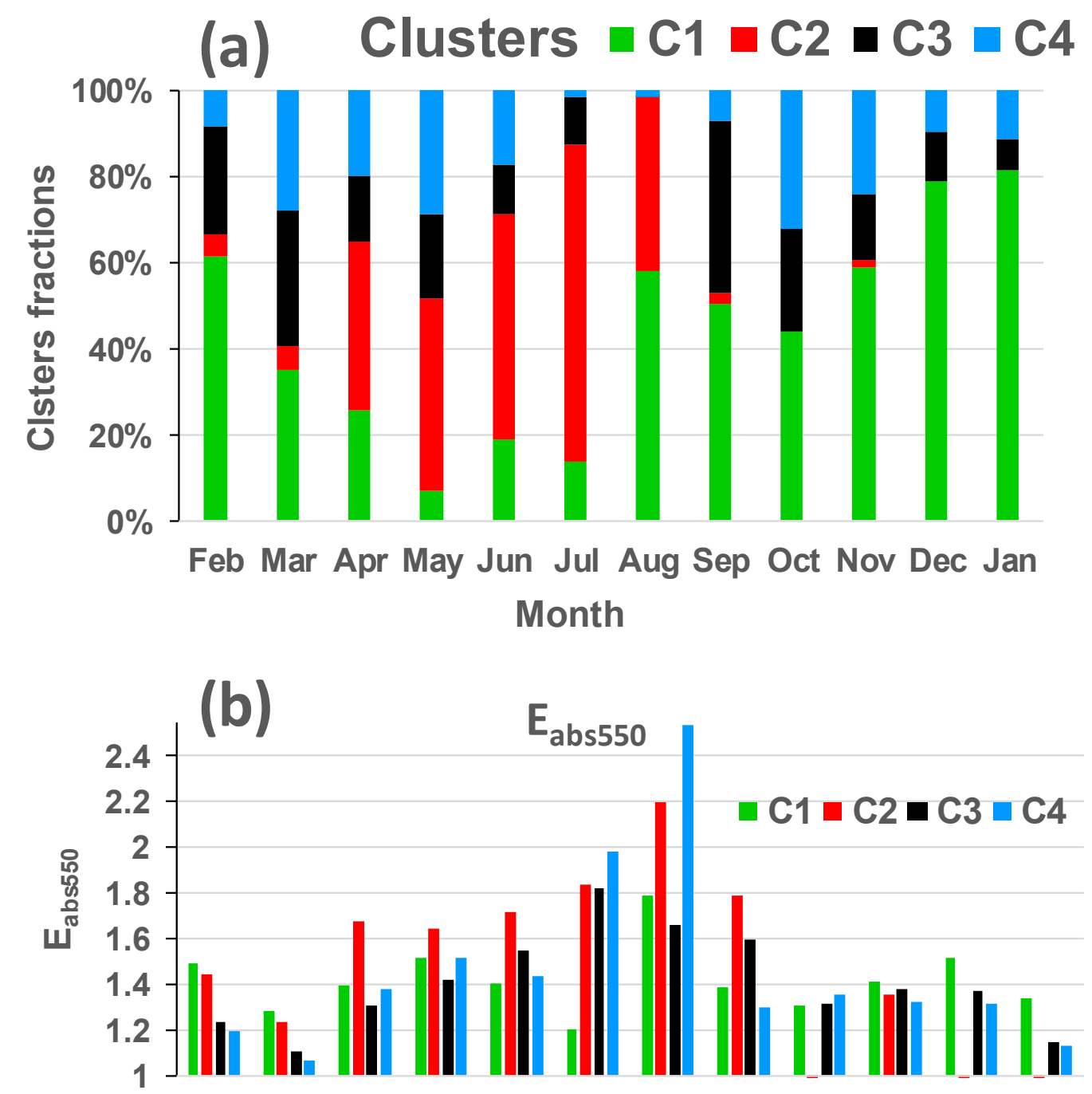

Feb Mar Apr May Jun Jul Aug Sep Oct Nov Dec Jan

Month

Figure S10. (a) Monthly contribution of each cluster. (b) Monthly Eabs550 of each cluster. 


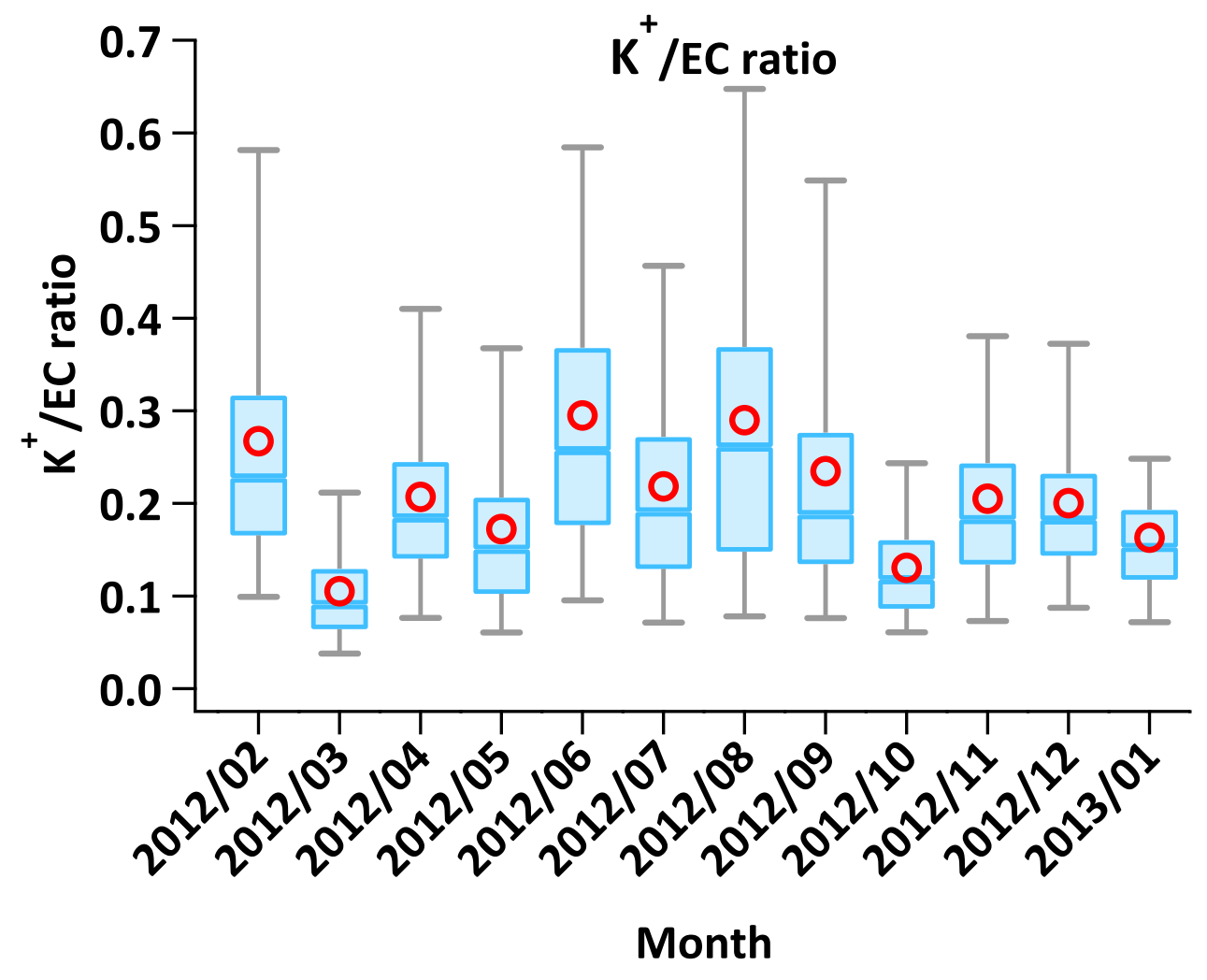

Figure S11. Monthly variations of $\mathrm{K}^{+} / \mathrm{EC}$ ratio from $2012 \mathrm{Feb}$ to 2013 Jan at NC site. 

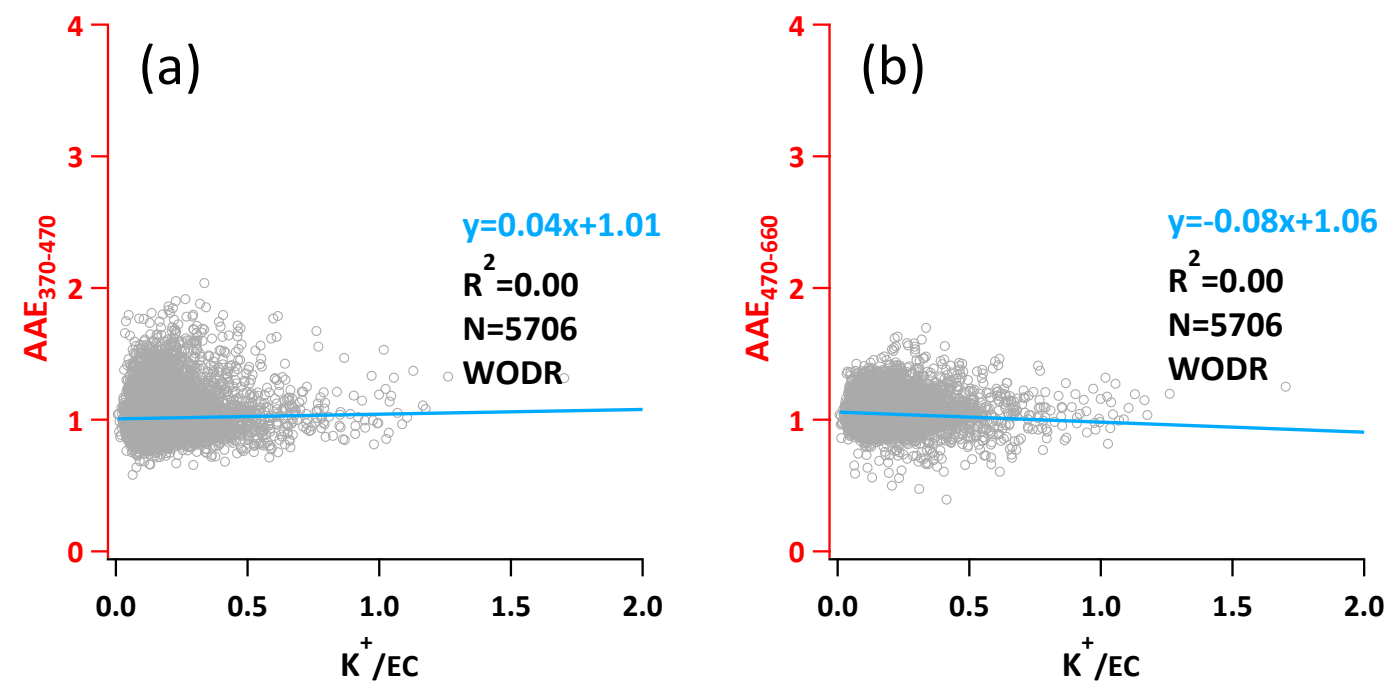

Figure $\mathrm{S} 12$. Correlations of $A A E$ with $\mathrm{K}^{+} / E C$ ratio (biomass burning indicator). (a) $A A E$ from 370 $-470 \mathrm{~nm}$. (b) AAE from $470-660 \mathrm{~nm}$. 


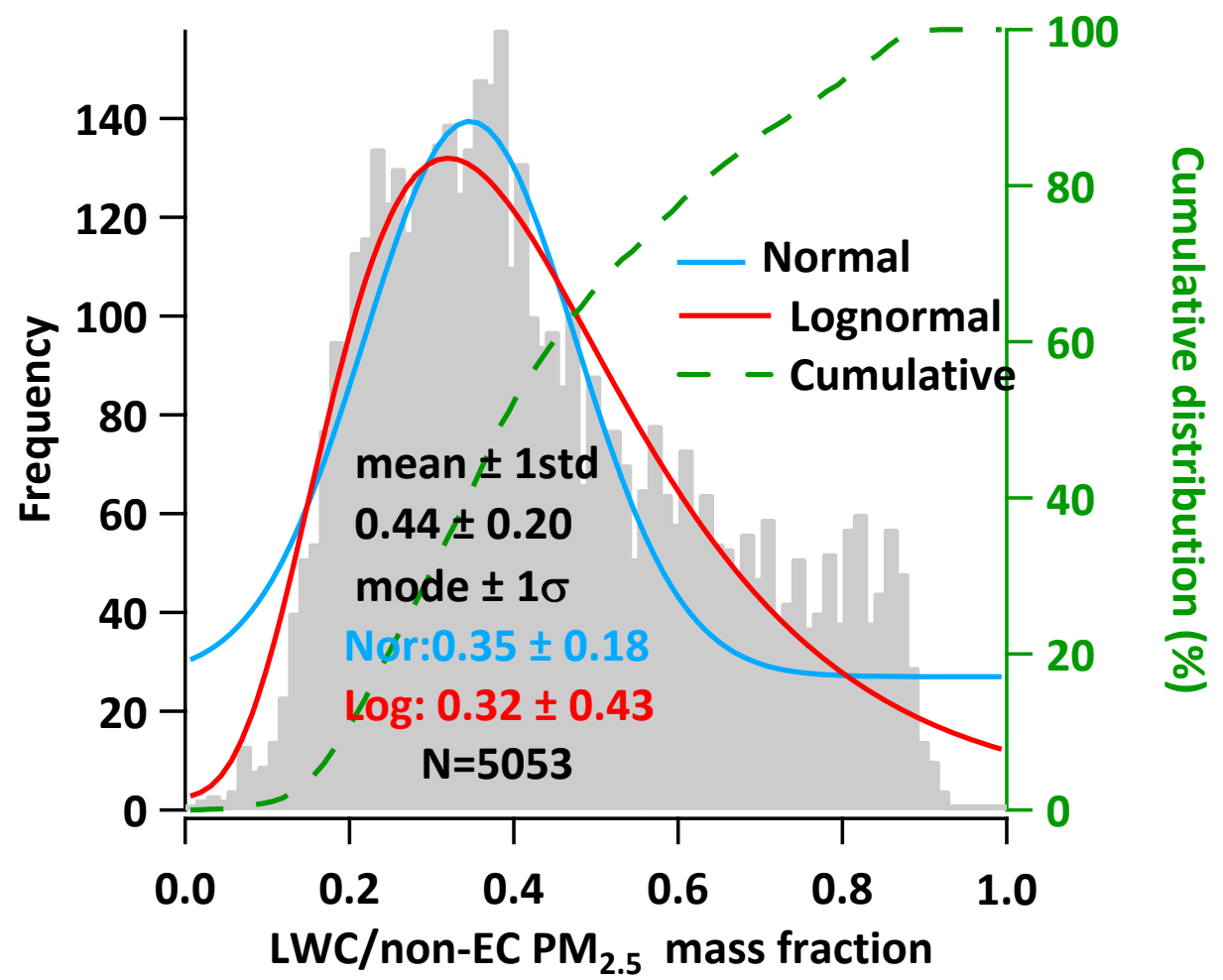

Figure S13. Annual frequency distribution of LWC/non-EC PM2.5 mass fraction. 


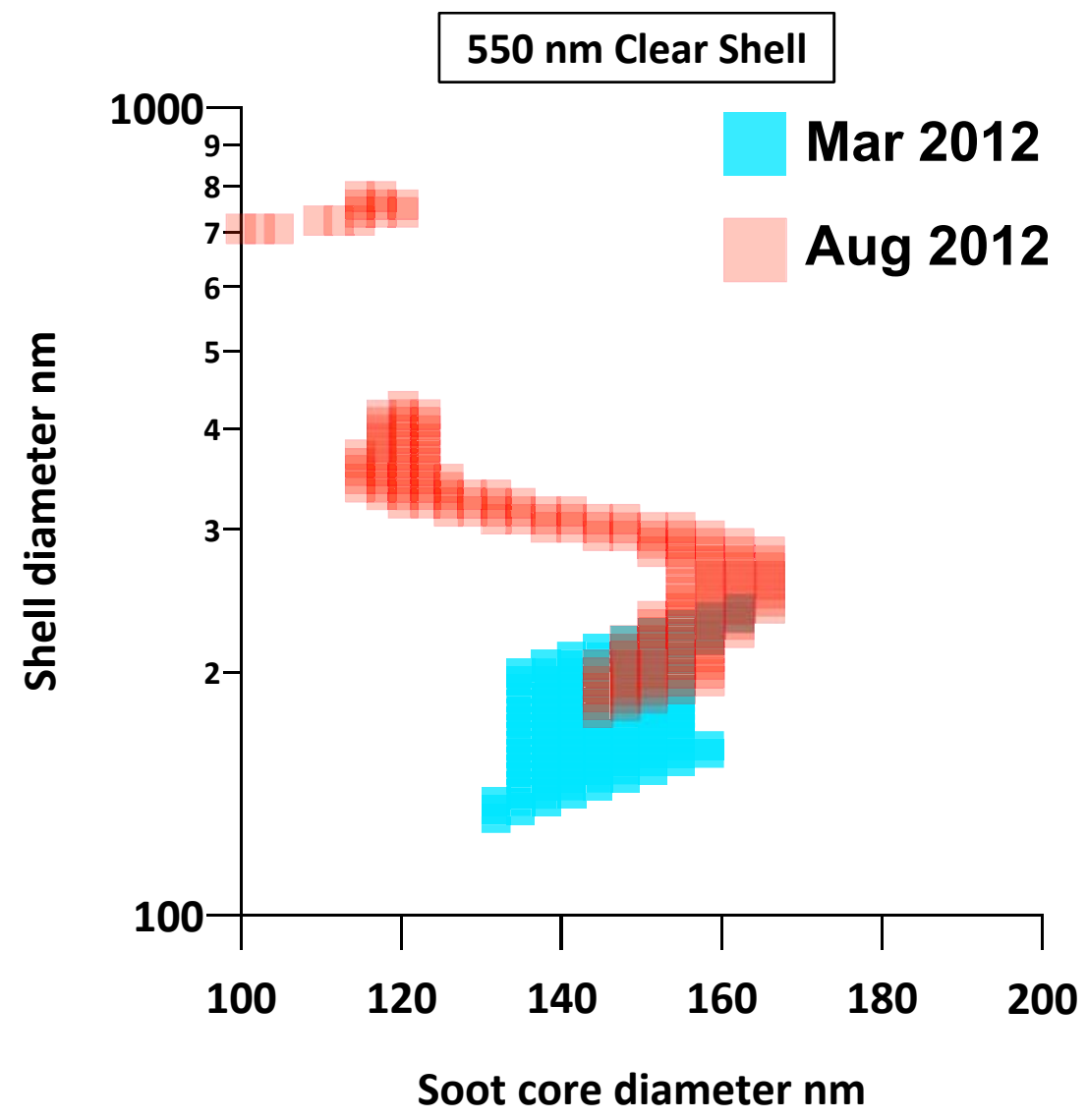

Figure $S 14$. Size range of soot particles constrained by $E_{a b s}$ and $A A E_{470-660}$ from measurements. 

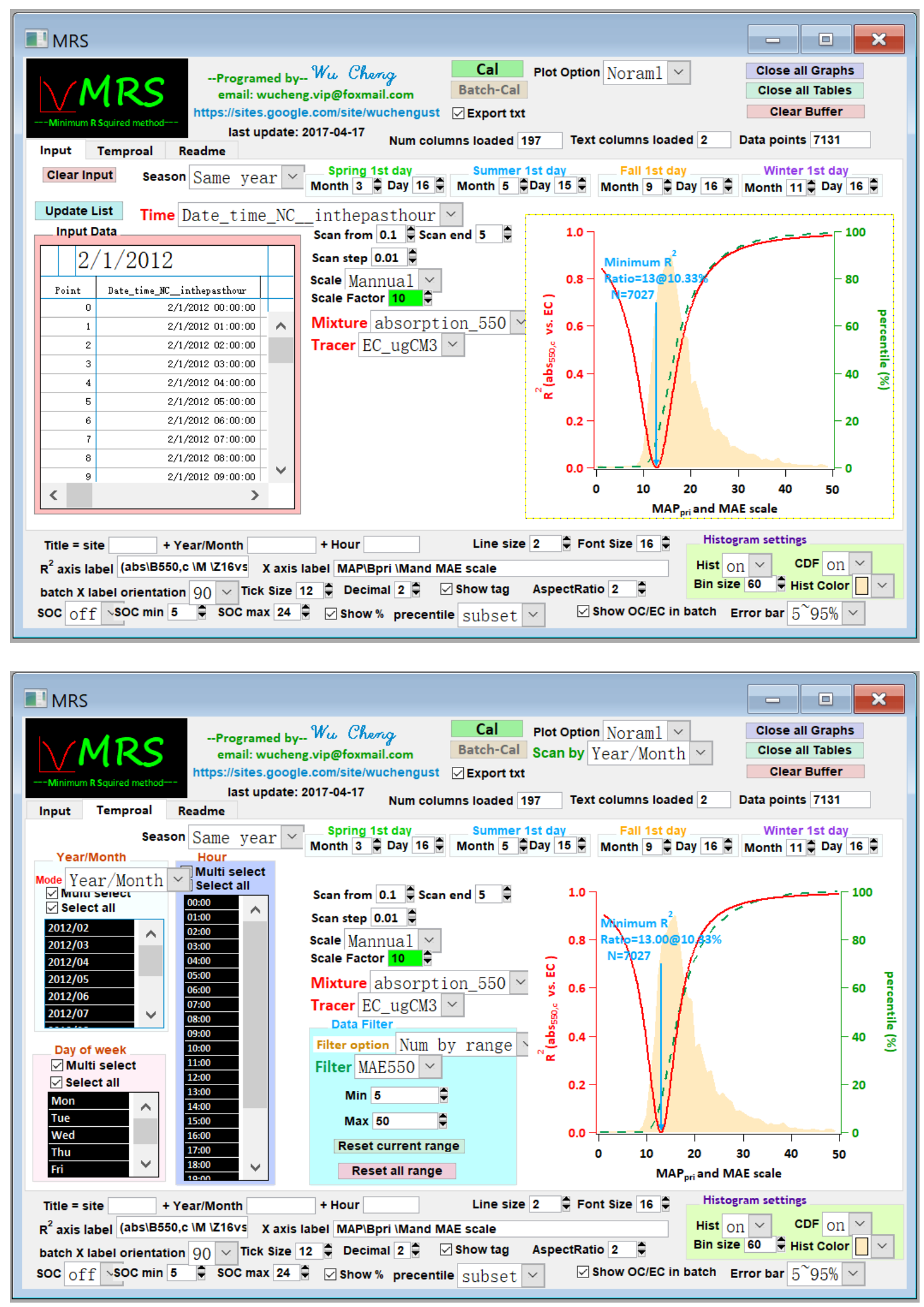

Figure S15. MRS program written in Igro Pro (WaveMetrics, Inc. Lake Oswego, OR, USA). Available from https://sites.google.com/site/wuchengust. 

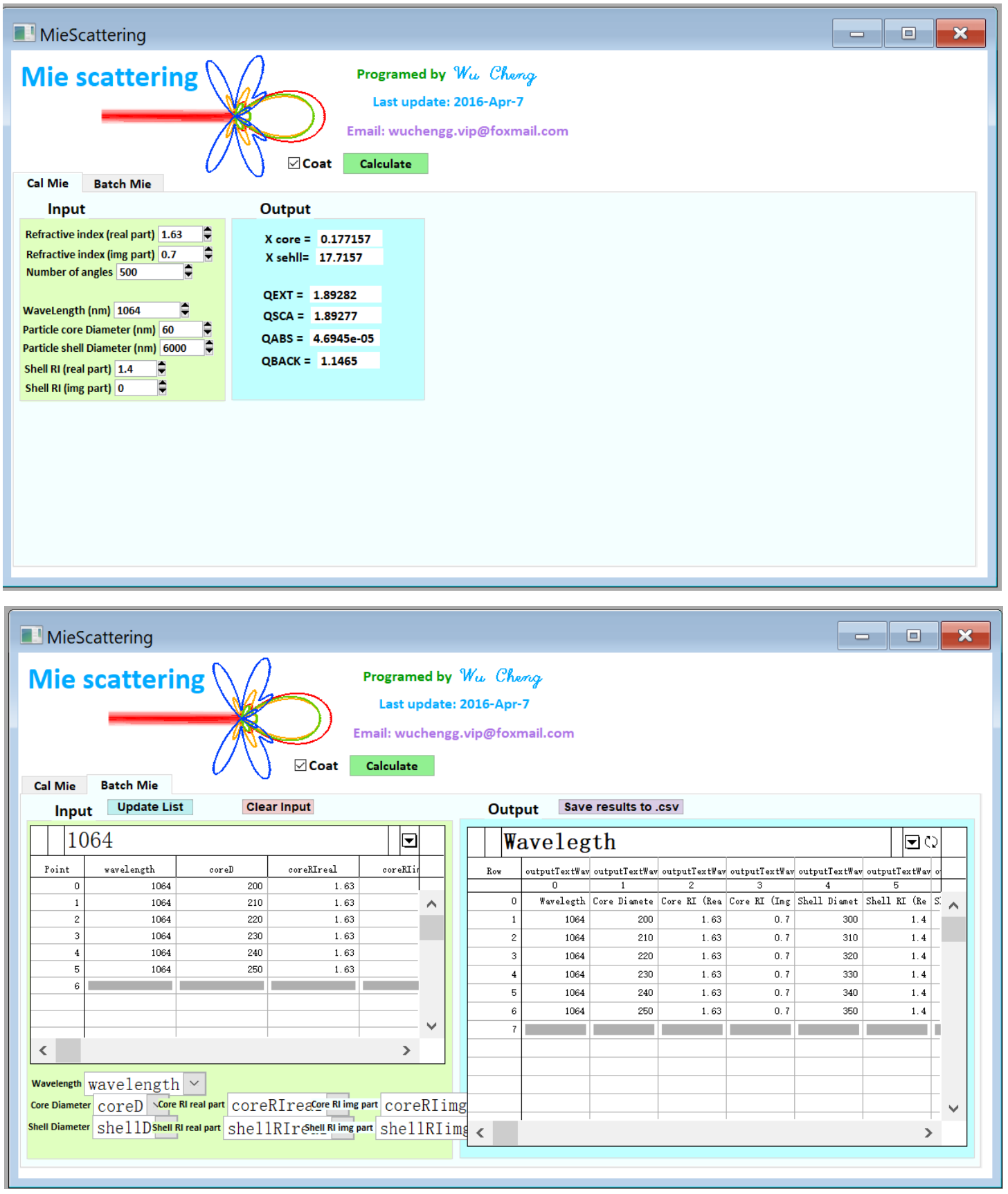

Figure S16. Mie program written in Igro Pro (WaveMetrics, Inc. Lake Oswego, OR, USA). Available from https://sites.google.com/site/wuchengust. 


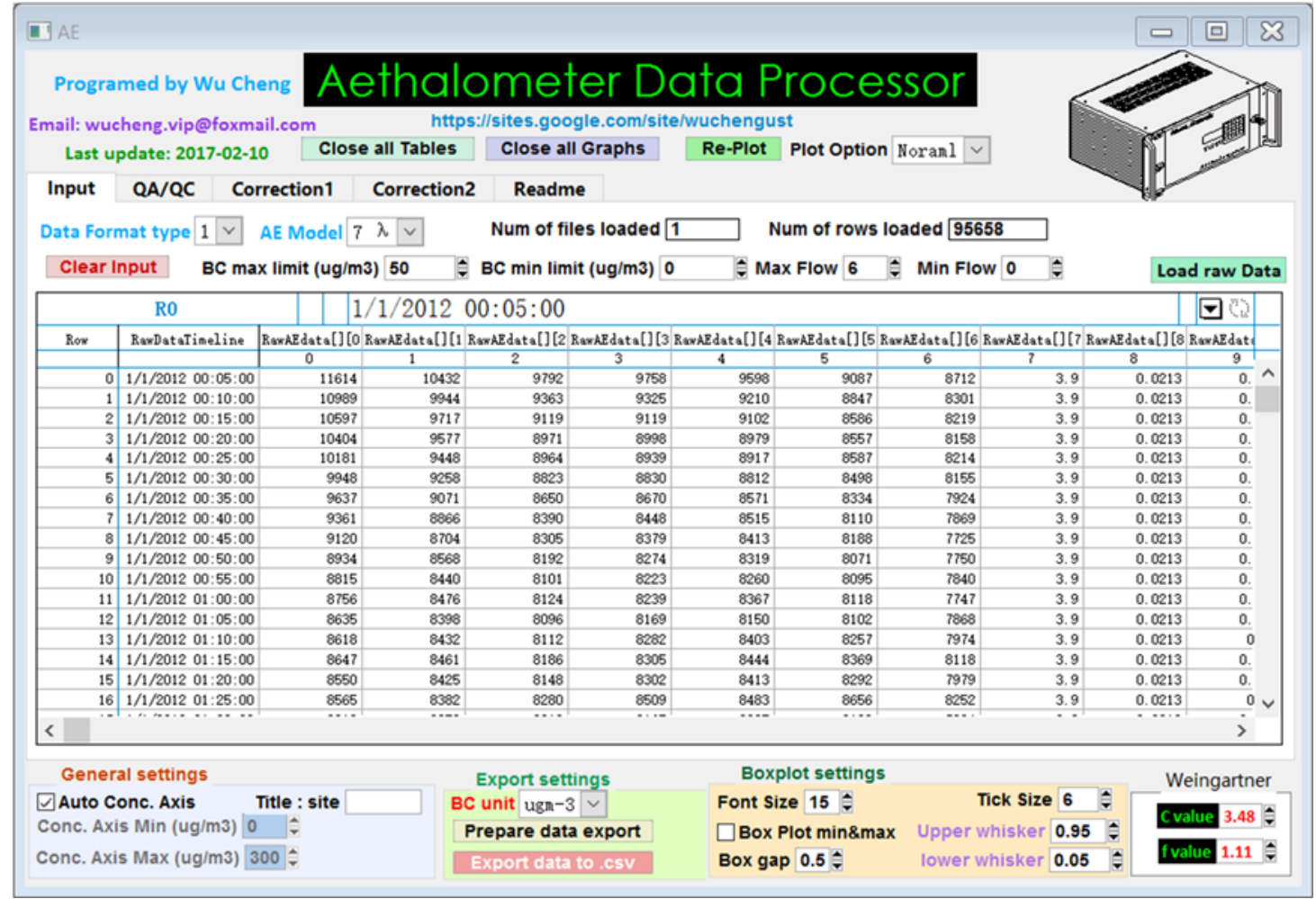

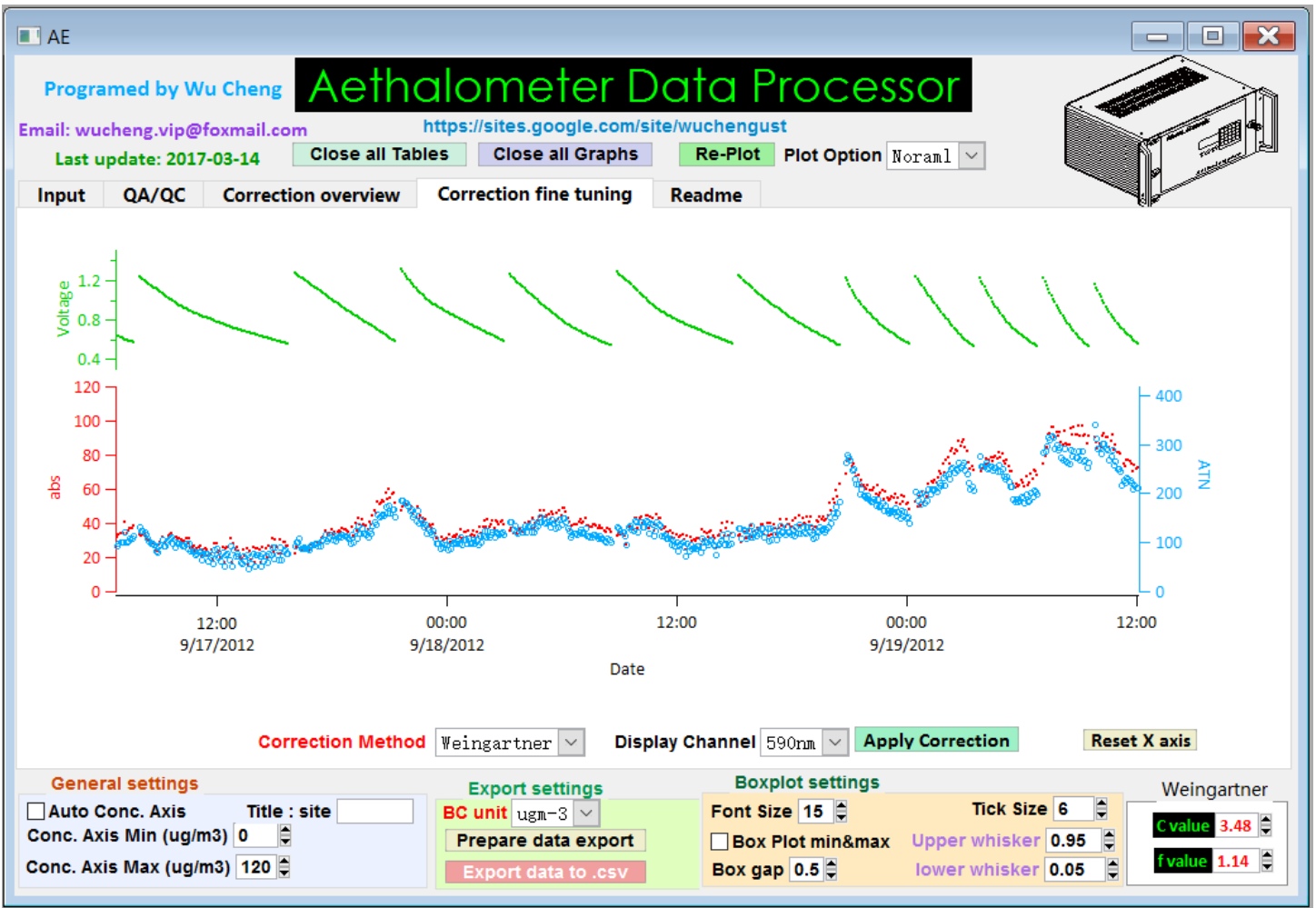

Figure S17. Aethalometer data processing program written in Igro Pro (WaveMetrics, Inc. Lake Oswego, OR, USA). Available from https://sites.google.com/site/wuchengust. 

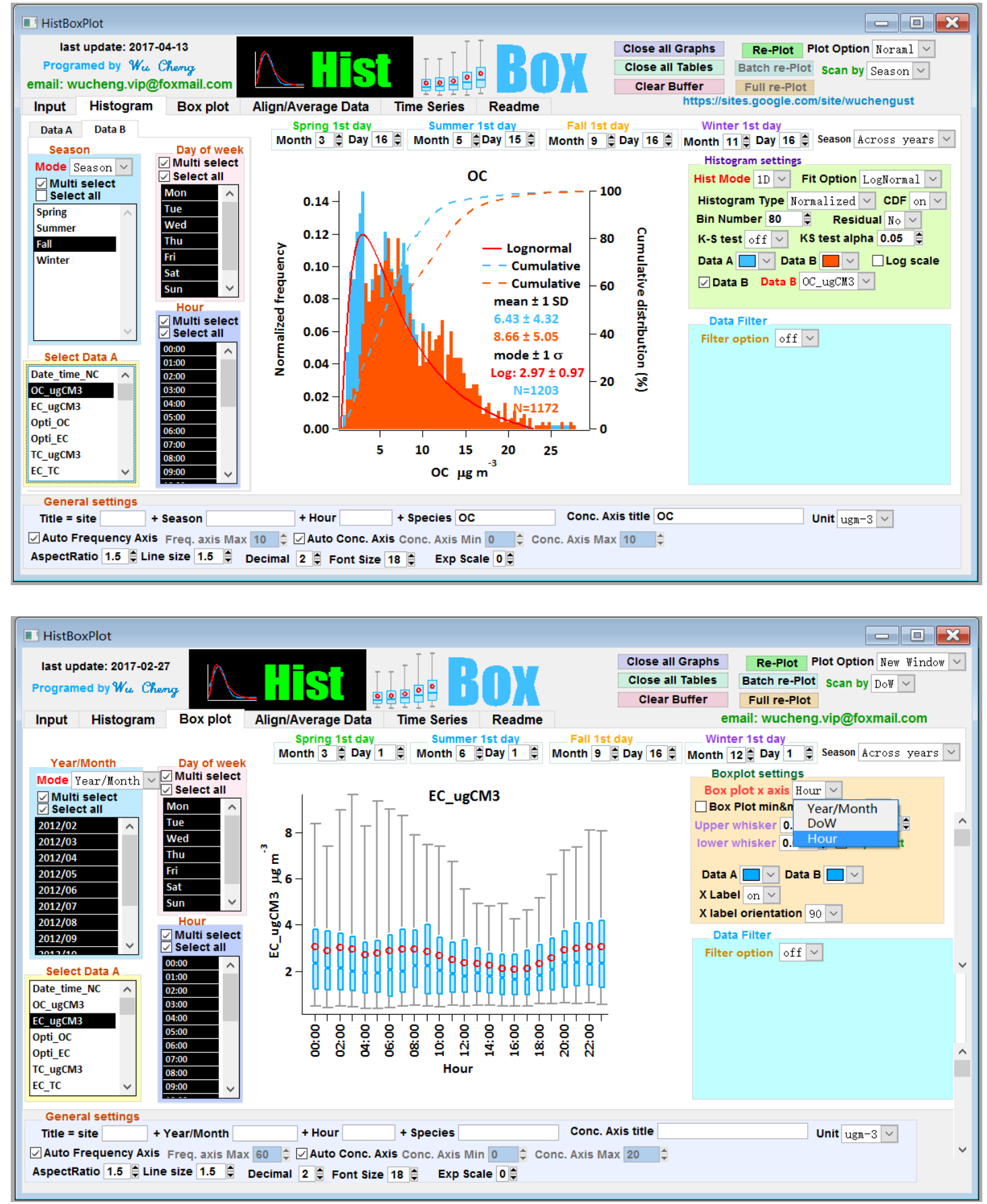

Figure S18. Histbox program written in Igro Pro (WaveMetrics, Inc. Lake Oswego, OR, USA). Available from https://sites.google.com/site/wuchengust. 

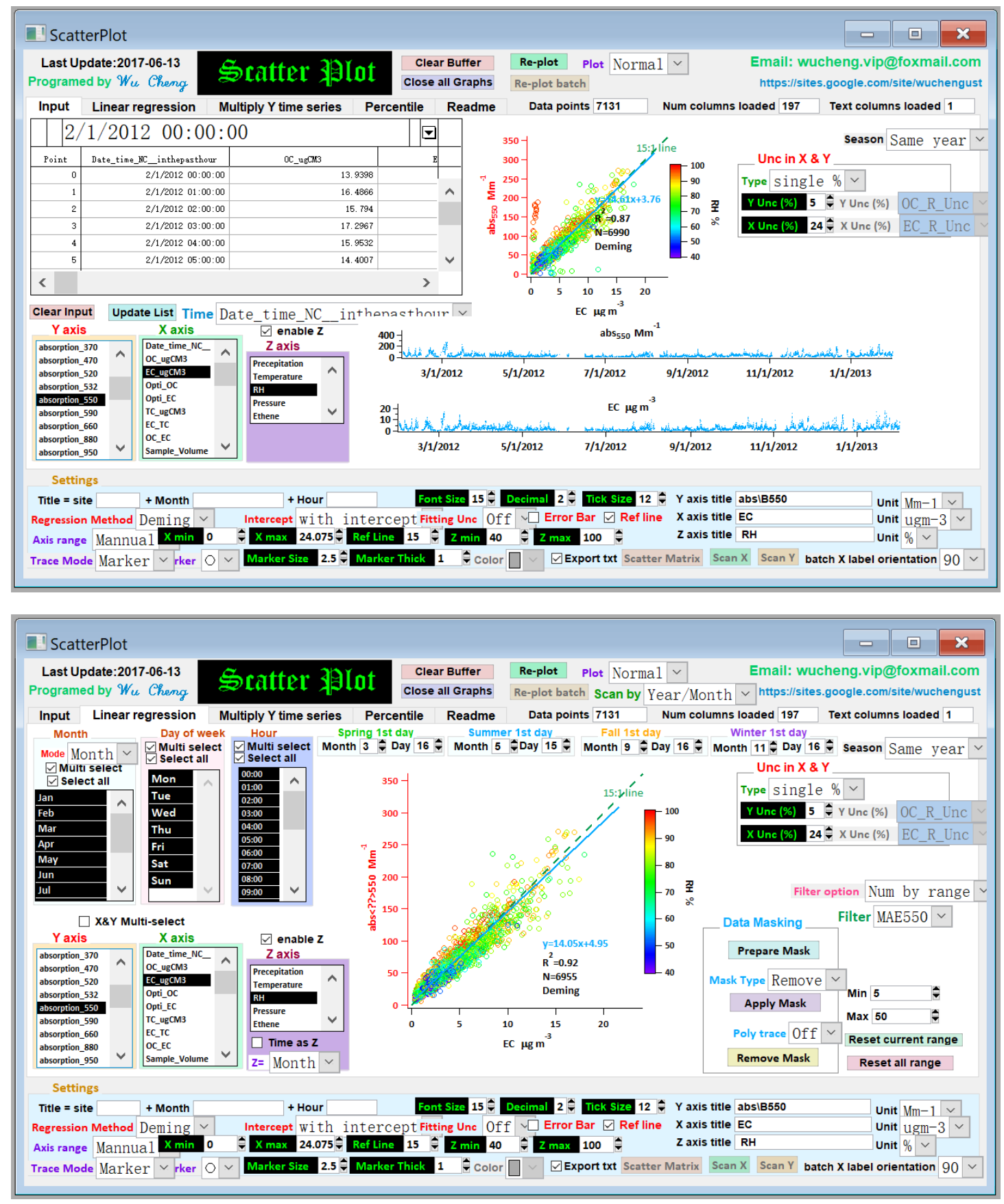

Figure S19. Scatter plot program written in Igro Pro (WaveMetrics, Inc. Lake Oswego, OR, USA). Available from https://sites.google.com/site/wuchengust. 